\title{
Growth Cone 3-D Morphology is Modified by Distinct Micropatterned Adhesion Substrates
}

\author{
Mirko Messa, Claudio Canale*, Emanuele Marconi, Roberto Cingolani, Marco Salerno, and Fabio Benfenati
}

\begin{abstract}
The development, connectivity, and structural plasticity of neuronal networks largely depend on the directional growth of axonal growth cones (GCs). The morphology and 3-D profile of axons and GCs of primary hippocampal neurons, grown onto glass surfaces coated with poly-D-lysine (PDL) and micropatterned with stripes of the adhesion molecule $\mathrm{L} 1$ by using the indirect microcontact printing, were investigated. Neurons were fixed at early stages (one to seven days) of in vitro development prior to synapse formation, and analyzed by fluorescence and atomic force microscopy. The latter technique allowed us to investigate the 3-D morphology of the GCs, and detect their morphological rearrangements during axon outgrowth and during contact with the underlying substrate. We found that axons decreased their height-to-width ratio over development in culture, and that this value became particularly low when the axon and the GC proceeded onto a surface containing attracting cues such as $\mathrm{L} 1$ with respect to GCs growing onto a nonspecific adhesion substrate such as PDL. Along with this shape change of the axons, GCs lying onto L1 tracks displayed a flattened shape, ideal for sensing and progression, whereas GCs onto areas of nonspecific adhesion displayed more prominent shapes and steeper edges.
\end{abstract}

Index Terms-Atomic force microscopy (AFM), cell-adhesion molecules, growth cones (GCs), microcontact printing $(\mu \mathrm{CP})$, primary neurons.

\section{INTRODUCTION}

D URING development, neurons send out axons to connect with appropriate targets cells and form synaptic connections. Each axon follows a specific pathway due to the sensorymotor properties of a high-motile structure localized at its distal tip, called the growth cone (GC). The GC is capable of detecting positive as well as negative environmental cues, and

Manuscript received October 28, 2008. First published April 10, 2009; current version published July 31, 2009. The work of F. Benfenati was supported by the Ministry of the University and Research Grants under Grant PRIN 2006, by the Compagnia di San Paolo, by the Telethon-Italy (GCP05134), and by Fondazione Pierfranco and Luisa Mariani grants. M. Messa and C. Canale contributed equally to this work. Asterisk indicates corresponding author.

M. Messa is with the Department of Experimental Medicine, Section of Physiology, University of Genova and Istituto Nazionale di Neuroscienze, Genova 16132, Italy, and also with the Department of Neuroscience and Brain Technologies, The Italian Institute of Technology, Genova 16163, Italy.

${ }^{*} \mathrm{C}$. Canale is with the Nanobiotechnology Platform, The Italian Institute of Technology, 16163 Genova, Italy (e-mail: claudio.canale@iit.it).

E. Marconi is with the Department of Experimental Medicine, Section of Physiology, University of Genova and Istituto Nazionale di Neuroscienze, Genova 16132, Italy, and also with the Department of Neuroscience and Brain Technologies, The Italian Institute of Technology, Genova 16163, Italy.

R. Cingolani and M. Salerno are with the Nanobiotechnology Platform, The Italian Institute of Technology, Genova 16163, Italy.

F. Benfenati is with the Department of Experimental Medicine, Section of Physiology, University of Genova and Istituto Nazionale di Neuroscienze, Genova 16132, Italy, and also with the Department of Neuroscience and Brain Technologies, The Italian Institute of Technology, Genova 16163, Italy.

Digital Object Identifier 10.1109/TNB.2009.2019109 to respond with directional growth, turning, or collapse [1]. Sensing the extracellular environment and adhering to the substrate is performed in the GC by different actin-rich structures, namely filopodia and lamellipodia, respectively [1]-[3]. Filopodia, in particular, interact through surface receptors with specific guidance factors such as diffusible molecules [4], cell-adhesion proteins [5], and extracellular matrix proteins [6], [7], thus controlling axonal elongation and guidance [8]. The GC elongation or retraction is associated with polymerization and depolymerization of actin, respectively, in a process known as actin treadmilling [9]-[12].

Studies in vitro of the GC behavior performed by classical culture techniques are often problematic due to the random organization of neurons even in low-density cultures [13]-[15]. Therefore, it is convenient to work on substrates with appropriately patterned adhesion and guidance materials. Furthermore, the presence of both attractive and repulsive areas on the same surface is necessary to confine both the position of the soma and the elongation of the neurites, and thus, provide a higher control on network organization [13], [16]-[18]. Over the past few years, an increasing number of surface modification techniques for biomolecular patterning have been developed, based on either photochemical methods [19], laser ablation of self-assembled monolayers [20], or microcontact printing ( $\mu \mathrm{CP}),[21]$, [22]. The latter technique, in particular, has spread in most applications, even if in some cases the use of $\mu \mathrm{CP}$ to pattern proteins or other biologically active molecules is difficult due to denaturation and consequent loss of function of the patterned protein during the drying required after inking of the elastomeric stamp [22], [23]. To overcome such limitations, an indirect $\mu \mathrm{CP}$ procedure has been developed, in which the active biological molecule fused to a tag is bound to a microprinted dry-resistant protein, acting as a bridge [24].

One of the best candidates for neuron as substrate-adhesion agent is L1 protein, a member of the immunoglobulin superfamily of cell-adhesion molecules [25]. L1 shows a typical extracellular region with six Ig-like domains and five fibronectin-type III (FNIII) domains, a single-pass transmembrane region, and an intracellular domain involved in intracellular signaling [26]. L1 is mainly expressed on the axonal surface [27], [28] where it binds in trans other L1 molecules expressed on the surface of other axons [29], [30]. L1 is not only one of the elements involved in clutch formation during filopodia probing, but it is also directly involved in the regulation of actin dynamics through interactions between its intracellular domain and actin binding proteins in the GC [31].

We have applied indirect $\mu \mathrm{CP}$ to pattern L1 and investigate the effect of such patterning on the growth of neurons on glass substrates. In order to obtain an active L1 pattern, a background 
pattern of protein A was generated to capture L1 expressed as a fusion protein with the protein A-binding immunoglobulin fragment $\mathrm{Fc}$ [32].

We have focused on the morphology of growing neurons, and particularly, on the environmental-dependent changes in GC morphology by using atomic force microscopy (AFM), a well-known technique in the study of cells in biocompatible environment [33]. The studies of neurons using AFM have often focused on the morphology of neuronal compartments, such as soma or dendritic spines [34]-[37]. Attempts to characterize the stiffness of various neuronal compartments such as axons or spines by AFM have also been made [38]. Some AFM studies also investigated aspects of GC physiology, such as the influence of the probe interaction force on the GC height [39] or the reorganization of the GC membrane by localized dissection with the AFM tip [40]. However, only few studies have been carried out thus far on the 3-D morphology of the GCs, resulting in the observation of filopodia and lamellipodia of neurons grown on to poly-L-lysine (PDL) [41].

Until now, little is known about the effect that the growth in a "stimulating" environment may have onto the axon and GC. We have tried to understand how the adhesion protein affects the behavior and the rearrangement of the 3-D shape of GCs with respect to a nonphysiological adhesion factor such as PDL. The use of AFM allowed us to extend our knowledge on the spatial rearrangement of the GC during axonal outgrowth by showing that GCs decreased their height-to-width ratio over development in culture, and that this decrease was particularly marked when the axon and GC proceed onto a surface containing attracting cues such as the L1 adhesion molecule. As a result of this shape change, GCs lying onto L1 tracks displayed a flattened shape, ideal for sensing and progression, whereas GCs onto areas of nonspecific adhesion showed more prominent shapes and steeper edges.

\section{MATERIALS AND METHODS}

\section{A. Neuronal Cultures}

Primary hippocampal neurons were harvested from E18 embryonic mice. Briefly, hippocampi were isolated and incubated for $15 \mathrm{~min}$ with $0.125 \%$ trypsin. After trypsinization, tissues were rinsed in plating medium (Neurobasal containing 10\% fetal bovine serum (FBS); Gibco-Invitrogen, Carlsbad, CA) and mechanically dissociated through a fire-polished Pasteur pipette. Neurons were seeded in plating medium on micropatterned coverslips at a density of 75-100 cells $/ \mathrm{mm}^{2}$ and incubated with $5 \%$ $\mathrm{CO}_{2}$ at saturated humidity. After $3 \mathrm{~h}$, to allow for cell attachment, the medium was replaced with serum-free Neurobasal supplemented with B-27 (Gibco-Invitrogen, Carlsbad, CA), and the cultures were followed daily for up to seven days in vitro (DIV).

\section{B. Preparation of L1-Fc Fusion Protein}

The plasmid encoding for the entire extracellular domain of L1 fused to the $\mathrm{Fc}$ region of human $\mathrm{IgG}$ under the control of the cytomegalovirus (CMV) promoter was obtained from Dr. P. Doherty (Guy's Hospital, London, U.K.) [42]. The plas- mid was transferred into COS-7 cells using diethylaminoethyl (DEAE) dextran, and cells were grown in advanced Dulbecco's modified eagle medium (DMEM) supplemented with 10\% FBS (Gibco-Invitrogen, Carlsbad, CA). The recombinant protein secreted into the medium was harvested and purified following the method described by Simmons [43]. Briefly, transfected COS-7 medium was gently spinned in order to remove cell debris, protein A-sepharose (Sigma-Aldrich, St. Louis, MO) was added to a final concentration of $10 \mathrm{mg} / \mathrm{mL}$ and gently stirred overnight at $4{ }^{\circ} \mathrm{C}$. The swollen beads were then poured into a column and abundantly washed with $1 \times$ phosphate buffered saline (PBS). After loading and washing the column, the bound L1-Fc was eluted by $100 \mathrm{mM}$ glycine- $\mathrm{HCl}, \mathrm{pH} 2.7$, and each eluted fraction of $0.5 \mathrm{~mL}$ was immediately neutralized by the addition of 50- $\mu \mathrm{L} 1 \mathrm{M}$ Tris-HCl, pH 9.0. Fractions containing the eluted recombinant protein were identified by sodium dodecylsulfate polyacrylamide gel electrophoresis (SDS-PAGE) on 7.5\% acrylamide gels and Coomassie brilliant blue staining. Integrity of the purified L1-Fc fusion protein was verified by using Western blotting with anti-L1 and anti-Fc antibodies (Sigma-Aldrich, St. Louis, MO). Protein concentration was determined using a Bradford assay kit (Bio-Rad Laboratories, Hercules, CA).

\section{Preparation of Polydimethyl Siloxane (PDMS) Stamps}

Silica master stamps were produced by means of standard radiation lithography techniques and wet etching at MDM-INFM Laboratories (Agrate Brianza, Milan, Italy). The silicon surface $\left(1 \mathrm{~cm}^{2}\right.$ area) was patterned with a square grid of $5-\mu \mathrm{m}$-wide lines spaced by $100 \mu \mathrm{m}$. PDMS stamps were casted from the masters, using Sylgard 184 silicone elastomer (Dow Corning, Midland, MI). The elastomer was combined with the curing agent in a 10:1 ratio, poured onto the masters, and degassed under vacuum to remove air bubbles. After $12 \mathrm{~h}$ at $120^{\circ} \mathrm{C}$, PDMS stamps were carefully removed and stored in double deionized water for $>24 \mathrm{~h}$ before use, in order to increase the stamp hydrophilicity and provide the best possible ink wetting and transfer thereof.

\section{Indirect $\mu C P$ of $L 1-F C$}

Glass coverslips (18 $\mathrm{mm}$ in diameter) were cleaned in concentrated nitric acid [44], coated with a layer of $0.1 \mathrm{mg} / \mathrm{mL}$ PDL (Sigma-Aldrich, St. Louis, MO) in deionized water, and micropatterned as follows. The PDMS stamps were sterilized in a $70 \%$ ethanol bath under ultrasonication for $10 \mathrm{~min}$, coated with $10 \mu \mathrm{g} / \mathrm{mL}$ protein A (Sigma-Aldrich, St. Louis, MO) or Alexa ${ }^{546}$-protein A (Molecular Probes-Invitrogen, Carlsbad, $\mathrm{CA}$ ) in water for $5 \mathrm{~min}$, and carefully dried under a $\mathrm{N}_{2}$ stream. The stamps were then placed onto the coverslips, kept in contact with them for $5 \mathrm{~min}$ under a constant pressure of $40 \mathrm{~g} / \mathrm{cm}^{2}$, and finally removed (see Fig. 1). The micropatterned coverslips were washed in water, dried under $\mathrm{N}_{2}$, and treated overnight with $1 \mu \mathrm{g} / \mathrm{mL} \mathrm{L} 1-\mathrm{Fc}$ fusion protein in $1 \times$ PBS. After washing with $1 \times$ PBS, the patterned substrates were placed in the plating medium for $2 \mathrm{~h}$ before the addition of dissociated neurons. 

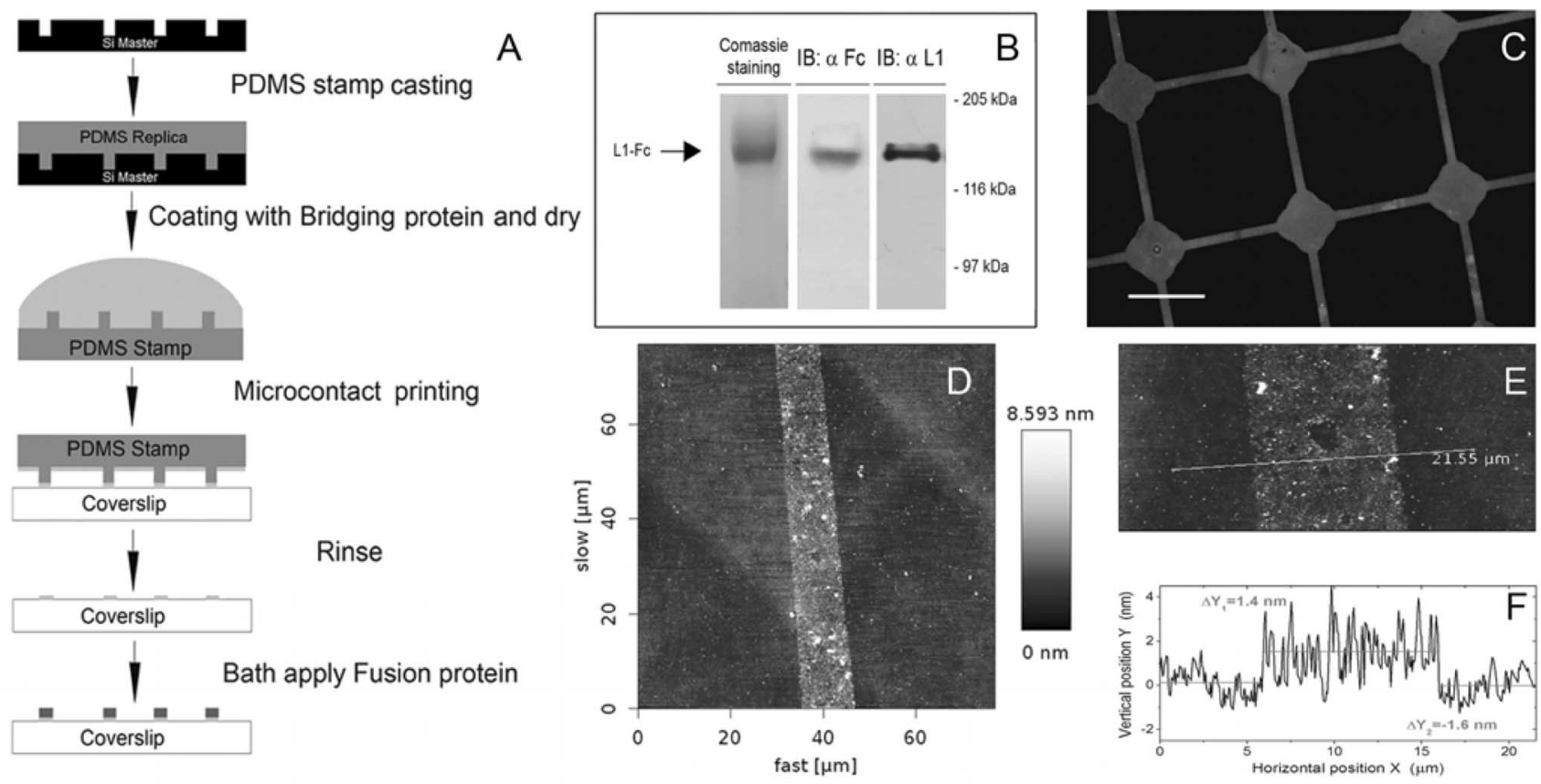

Fig. 1. Indirect $\mu \mathrm{CP}$ of protein stripes. (a) Sketch of the sequential processing steps of the $\mu \mathrm{CP}$ technique. Silicon masters were used to cast PDMS stamps. The stamps were inked with Alexa ${ }^{546}$-protein A and dried. The coated stamps were then contacted to coverslips and removed, obtaining a layer of micropatterned protein A (light gray). The coverslip was incubated with L1-Fc, which bound directly to protein A (dark gray). (b) Protein integrity control of purified L1-Fc. Affinity purified L1-Fc was subjected to SDS-PAGE followed by either (left) Coomassie blue staining, (middle) Western blotting with antibodies against Fc, or (right) L1. (c) Large-view fluorescence microscopy image of a typical pattern of fluorescently labeled protein A. Scale bar, $100 \mu \mathrm{m}$. (d) Midview (77 $\mu \mathrm{m} \times 77 \mu \mathrm{m}$ scan, scale bar $20 \mu \mathrm{m}$ ) of AFM images . (e) Closeview (30 $\mu \mathrm{m} \times 15 \mu \mathrm{m}$ scan) of AFM images of the pattern reported in (d). (f) Profile showing the protein stripe cross section taken at the transversal line traced in (e). The resulting step height is $\sim 1.5 \mathrm{~nm}$.

\section{E. Optical Imaging of Neuronal Cultures}

Live neurons were followed during early stages of development in culture (one to seven DIV) using phase contrast microscopy. Samples were fixed with $4 \%$ paraformaldehyde in $1 \times$ PBS for $10 \mathrm{~min}$, and then rinsed with $1 \times$ PBS. Neurons in the micropatterned networks were stained using an anti- $\beta$ III tubulin monoclonal antibody (Millipore, Billerica, MA) or tetramethylrhodaminoisothiocyanate (TRITC) conjugated phalloidin (Molecular Probes-Invitrogen, Carlsbad, CA). Fixed cells were permeabilized for $5 \mathrm{~min}$ with $0.1 \%$ Triton X-100, $0.1 \%$ $\mathrm{NaN}_{3}$ in $1 \times$ PBS and blocked against nonspecific binding with $4 \% \mathrm{FBS}, 0.1 \% \mathrm{NaN}_{3}$ in $1 \mathrm{X}$ PBS for $30 \mathrm{~min}$. Cultures were rinsed with $1 \times$ PBS and incubated with primary anti- $\beta$ III tubulin antibody for $3 \mathrm{~h}$. After rinsing, TRITC-phalloidin was applied for $10 \mathrm{~min}$, followed by the application of Alexa ${ }^{488}$ goat antimouse IgG (Molecular Probes, Invitrogen, Carlsbad, CA) as secondary antibody for $45 \mathrm{~min}$. The cultures were finally rinsed with $5 \mathrm{mM}$ phosphate buffer and mounted on Mowiol (Molecular Probes, Invitrogen, Carlsbad, CA) on glass slides. Images were acquired using a Leica SP5 confocal microscope (Leica Microsystems, Wetzlar, Germany) and an Olympus IX71 fluorescence microscope equipped with the Cell Imaging System (Olympus, Tokyo, Japan). When fluorescence and AFM were combined, images were taken from the AFM setup [see Fig. 3(a) and (c)], the exact superimposition of the corresponding images was obtained by using the DirectOverlay routine of the AFM acquisition software (JPK Instruments, Berlin, Germany).

\section{F. AFM Imaging}

AFM measurements were performed using a JPK NanoWizard II system mounted on an inverted optical microscope Olympus IX71 equipped with $4 \times$ and $40 \times$ objectives. The fluorescence signal was excited through a Xenon cold fiber-guided fluorescence source X-Cite120 (EXFO, Mississauga, ON, Canada), and the images were acquired with a color charge-coupled device (CCD) camera (Imagingsource, Bremen, Germany) (see Fig. 3). All the experiments were performed on samples fixed as previously described. Tapping-mode AFM images were taken in liquid (PBS $1 \times$ ) in the constant-amplitude mode, using V-shaped silicon nitride cantilevers (Veeco, Santa Barbara, CA) with a typical spring constant of $0.32 \mathrm{~N} / \mathrm{m}$. The probe-sample interaction was minimized by continuously adjusting the set point during imaging. The scan rate was set between 0.2 and $1 \mathrm{~Hz}$. Height was collected simultaneously with the amplitude and phase signals in both trace and retrace directions. Images were line-fitted as required. Occasionally, isolated scan lines were removed.

\section{G. AFM Data analysis}

From the top-view images of the different sets of cultured neurons, height profiles of axons and axonal GCs were taken. We analyzed both the longitudinal and transverse profiles of the axons, in order to follow the axon geometry along its length and estimate fluctuations. A minimum number of seven transverse 
profiles per axon were measured along straight lines perpendicular to the axon axis in the region of interest.

Each axonal longitudinal profile has been taken along curved lines to follow the main axis of the respective axon and GC [see, e.g., Fig. 5(a)]. To measure the data points corresponding to curved longitudinal profiles from the AFM top-view height images, a dedicated software routine was developed in MATLAB (The MathWorks, Natick, MA). The criterion for the selection of the curved path was based on an automatic routine for finding the maximum local height around the central ridge of the axon. In few cases, this routine failed due to large and tall off-axis nonneuronal structures, the actual axon axial path was manually corrected to coincide with the most meaningful central profile.

In order to compare GC shapes, the heights of each profile were normalized to the mean height of the axon. On the contrary, the length ( $x$-axis) of the profiles was measured in real physical units (in micrometers) starting from the terminal end of the GC.

From the transversal height profiles of the axons [white lines in Fig. 5(a)], representative values of both height $h$ and width $w$ were extracted. For the width, the full-width at half maximum of the profiles was considered. The aspect ratio a.r. $=h / w$ at each profile were then calculated from these values, and the absolute error $e$ on the a.r. was estimated, after the theory of error propagation. Both a.r. and $e$ were averaged for each axon over the various positions. Finally, a.r. and $e$ were averaged for all the axons imaged at the same time in vitro and plotted as $\langle$ a.r. $\rangle \pm\langle e\rangle$.

From the longitudinal axon and GC profiles [black line in Fig. 5(a)], a qualitative assessment of the GC shape and a quantitative estimation of the GC heights were carried out. In the case of GCs with prominent profile, the GC domain was easily identified as the part of the axon termination that was significantly higher with respect to the mean axon height. In the absence of significant peaks in the GC profile, the GC extension was arbitrarily defined as the interval between 6 and $1 \mu \mathrm{m}$ from the distal end of the axon.

\section{RESULTS AND DISCUSSION}

\section{A. Morphology of the Microprinted Profiles of LI}

In order to avoid the loss of biological activity of directly micropatterned L1 adhesion protein, the indirect $\mu \mathrm{CP}$ technique was used. According to this technique, an intermediate molecule resistant to denaturation such as protein A was patterned on glass coverslips, which were subsequently incubated under physiological conditions with the purified L1-Fc chimeric protein [see Fig. 1(b)]. This procedure is believed and orientation toward the subsequently plated neurons. Protein A was microprinted on bare glass using a PDMS stamp casted from a silicon master with a grid-like pattern (see Fig. 1(a)]. The printing profiles visualized by fluorescently labeled protein A [see Fig. 1(c)] precisely and homogeneously reproduced the stamp pattern. Analysis by AFM [see Fig. 1(d)-(f)] revealed that the protein A stripes had a homogeneous surface, and were characterized by an average thickness of about $1.5 \mathrm{~nm}$ over the PDL-coated glass surface.
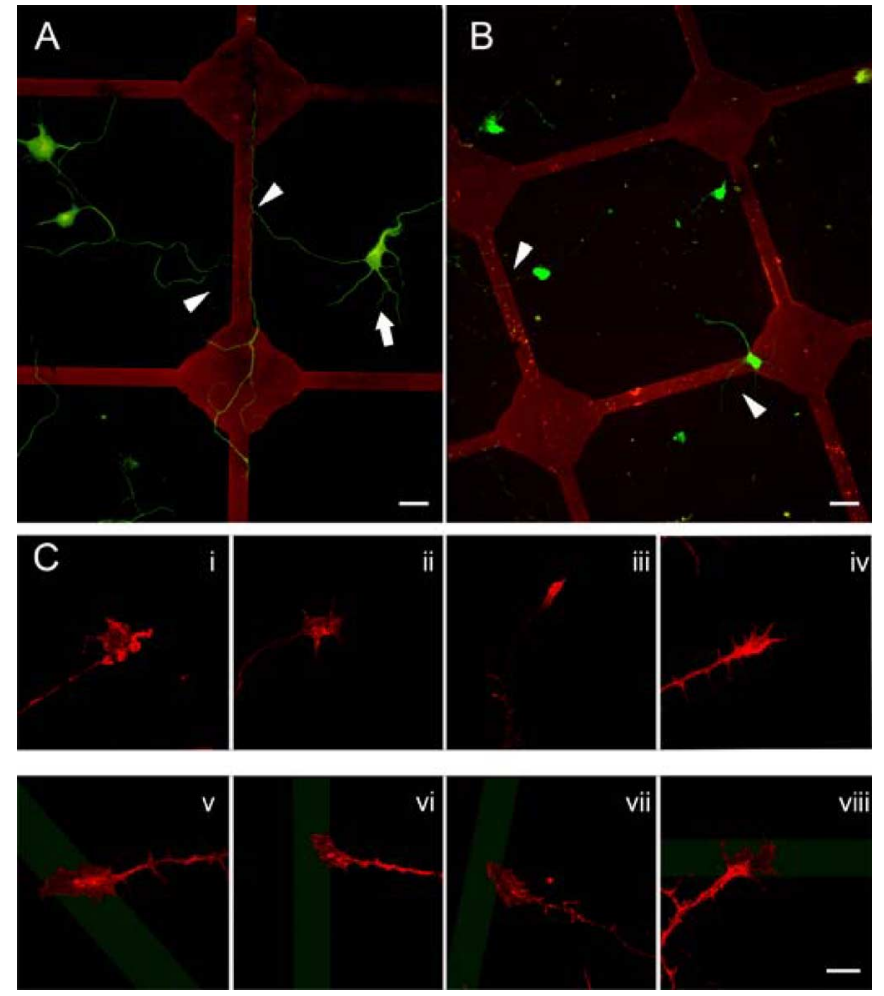

Fig. 2. Fluorescence images of neurons grown onto micropatterned L1-Fc. (a) and (b) Cultured neurons were fixed at seven DIV and labeled for $\beta$ IIItubulin. (a) Representative image of neurons grown on glass coverslips coated with PDL and subsequently patterned with L1-Fc (red grid pattern). Axons (arrowheads) preferentially moved along the L1-Fc stripes, while soma and dendrites (arrow) did not respond to L1 and grew on PDL-coated areas. (b) Negative control in which L1-Fc was omitted (only protein A was patterned). Axons (arrowheads) did not align with the pattern and randomly crossed the protein A tracks. (c) Gallery of high-resolution confocal images of axonal GCs, from four DIV cultured neurons, labeled for F-actin by TRIC-phalloidin (red). Axons and GCs that grew onto PDL-coated areas (i-iv) or on L1-Fc stripes (green; v-viii) display distinct shapes. Scale bars: $20 \mu \mathrm{m}$ (a, b); $5 \mu \mathrm{m}$ (c).

\section{B. Guided Axonal Growth on Protein Patterns}

We first analyzed the axonal guidance role of the patterned L1 and followed the 3-D profiles of axonal GCs by confocal microscopy [see Fig. 2(c)] as well as by combined epifluorescence/AFM technique (see Fig. 3). With respect to the large PDL-coated surfaces [black areas in Fig. 2(a) and (c)], axons were attracted by the L1-coated surfaces (red stripes), and once they had reached them, turned to align and grow along the tracks. The approach and the alignment of neuronal processes with the L1 stripes were also monitored by AFM imaging of neurons at various developmental stages in culture. Fig. 3(b) shows an AFM snapshot of an axonal GC at an early developmental stage (four DIV), which invaded the L1 stripe and turned right to align with the track. At later stages (14 DIV), neuronal processes identified by AFM and overlaid on the fluorescent image of the adhesion protein stripe (which was not very evident from the AFM height map) remain precisely confined to the L1-patterned profiles [see Fig. 3(c)]. When L1 was omitted during the indirect $\mu \mathrm{CP}$ procedure [see Fig. 2(b)] then no such alignment was observed, the axons grew with random orientation and crossed the protein A-coated tracks as if no pattern was 

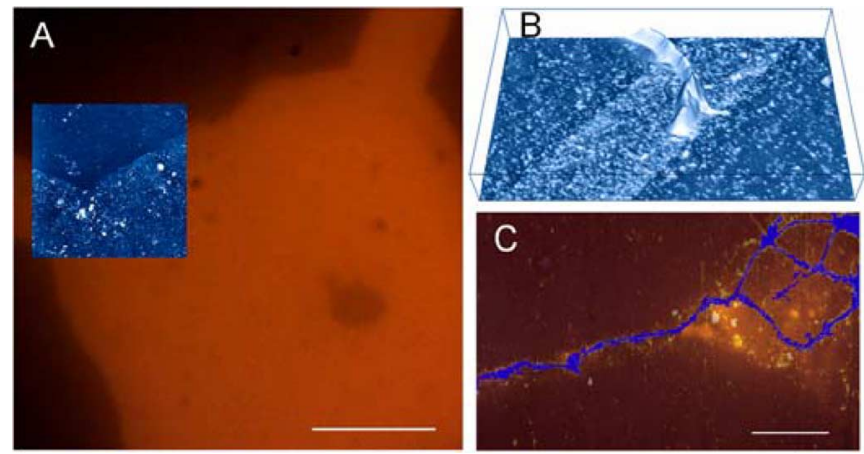

Fig. 3. Analysis of GC morphology by combined AFM-fluorescence microscopy. AFM features in blue, while fluorescence microscopy features in red. (a) Fluorescence image of Alexa ${ }^{546}$-protein A pattern with overlaid AFM topography (inset). (b) AFM 3-D topography of a young (four DIV) neuronal GC that reached an L1 stripe. ( $45 \mu \mathrm{m} \times 27 \mu \mathrm{m}$ scan.) (c) Fluorescence image of L1 pattern with overlaid AFM topography of mature (14 DIV) neurons. Scale bars: $20 \mu \mathrm{m}(\mathrm{a}) ; 10 \mu \mathrm{m}(\mathrm{b})$.
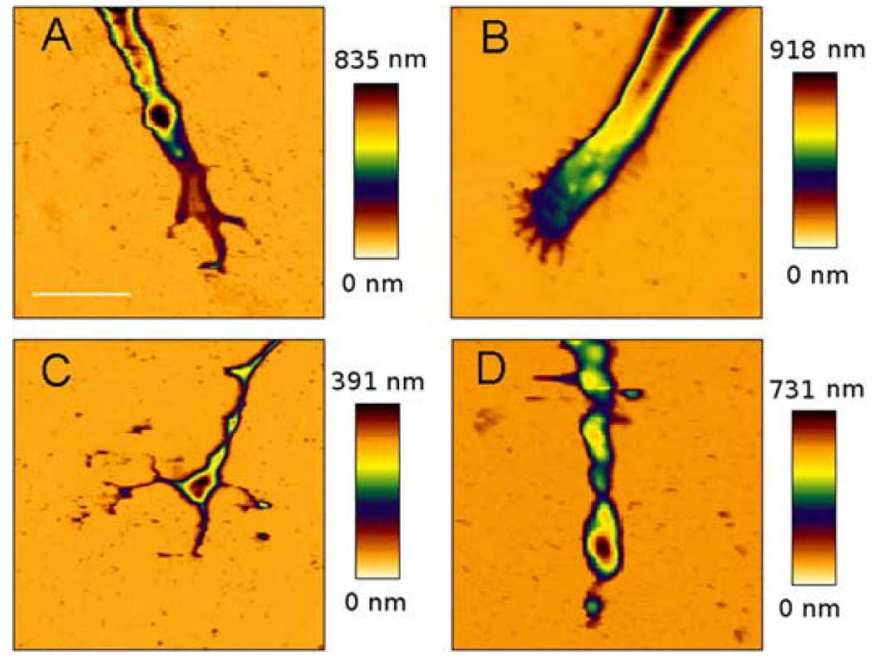

Fig. 4. Representative AFM images of axons and their terminal GCs. (a) and (b) Axons grown onto L1 stripes are characterized by flat, "open-hand"-shaped GCs. (c) and (d) Axons grown off the L1 stripes are characterized by high, "clenched-hand"-shaped GCs. Scale bar, $5 \mu \mathrm{m}$.

present. These observations confirm the specificity of the L1 effect and demonstrate the preservation of its biological activity during the microprinting procedure.

\section{Morphology of the GCs Growing Along Unpatterned or Patterned Surfaces}

We have studied the 3-D shape of axonal GCs as a function of the surrounding environment by means of AFM. We focused our AFM analysis on two distinct groups of GCs, namely those growing onto PDL surfaces, and those that reached the L1 stripes and started outgrowing onto them. From a series of sample images (Fig. 4), it appeared clear that the substrate strongly affected the gross GC morphology. Axonal GCs growing on top of the L1 stripes had an open and flat appearance [see Fig. 4(a) and (b)], whereas GCs moving out of the L1 stripes, in contact with the PDL-coated background surface, displayed a high and clenched shape [see Fig. 4(c) and (d)].
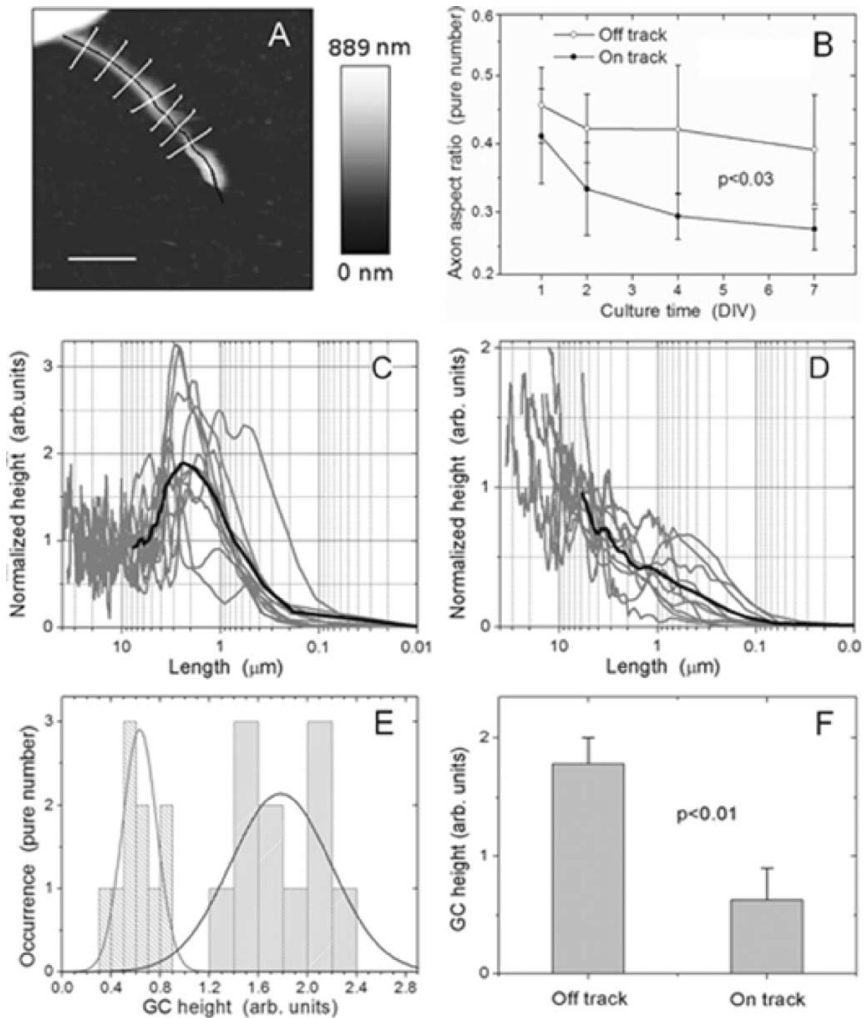

Fig. 5. Analysis of the axon height profiles from AFM topographical images. (a) Typical selection of the traces for determination of the longitudinal and transversal profiles. (b) Plot of the mean aspect ratios (height-to-width values) of the axons (excluding the GC) extracted from the transversal profiles, as a function of the developmental stage (from one to seven DIV) $\left(^{*}, p<0.05\right.$, one-way ANOVA). (c) and (d) Longitudinal axon profiles taken for 21 neurons whose axons were lying either off [(c), $n=11]$ or on $[(\mathrm{d}), n=10)$ the $\mathrm{L} 1$ pattern, respectively. (e) Distribution of the GC heights as obtained from the profiles shown in (c) and (d) for the two groups of off-track (right; thick bars) and on-track (left; thin bars) axons. Bin size was adapted to the distribution of GC height in each group. The continuous curves are Gaussian fits for the two groups. $(F)$ Mean heights and respective standard errors for the two populations of GCs as identified in (e) by the two Gaussian fits $\left({ }^{* *}, p<0.01\right.$, one-way ANOVA).

To quantitatively analyze these 3-D shape differences, we measured the height of the longitudinal GC profiles and the width of the transverse profiles along the axon and the GC [see Fig. 5(a)], and grouped the profiles into two groups, according to whether the respective axon $+\mathrm{GC}$ were observed to lie on or off the L1 stripes.

By measuring height and width of the axons in our samples, we found the tendency of the axons to become flatter with time of in vitro development, whereas their height remained relatively constant. As a result, the a.r. (see Materials and Methods) decreased as function of the developmental stage [about 10\%$20 \%$ from one to seven DIV, $n=7 / 17$ for experimental group, see Fig. 5(b)]. Interestingly, the a.r.s of axons laying on PDL and axons located on the L1 revealed a clearly different behavior. The axons on L1 stripes had a significantly lower a.r. (i.e. they were flatter than the axons on PDL), and the difference became more pronounced with time in vitro [see Fig. 5(b)].

The height profiles of the preterminal region of the axon and of the GC lying either off or on the L1 stripes [see Fig. 5(c)] were individually measured from several neurons $(n=21)$ at 
early developmental stages (one to seven DIV) and plotted as a function of the process length. The length of the imaged profiles (axon + GC) ranged from $\sim 6$ to $\sim 39 \mu \mathrm{m}$, due to the different growth of individual neurons in the same population and the culture time. The respective heights on top of the GC and the axon were also different and ranged from $\sim 200$ to $\sim 650 \mathrm{~nm}$ for the axons only.

In both groups, individual profiles were aligned on the basis of the distal edge of the GC, and were plotted in the same color (gray) to ascertain whether they shared a common profile pattern and were heterogeneous in shape. The mean profile for each group (termed "off-track" and "on-track," respectively) was plotted in black. Obviously, these profiles are only defined between $x=0$ and $x=L$, where $L$ is the length of the shortest profile. As shown in Fig. 5(c) and (d), the two groups of profiles indeed showed distinct characteristics: the off-track group of profiles was made of GCs with one major (and in some cases a few minor) height peaks, outstanding the mean axon level $(h=1)$. The on-track profiles, on the contrary, exhibited a progressively decrementing height toward the distal end (from left to right in the plot), with some fluctuations around this trend, but in the absence of height peaks. If the GCs are compared to little neuronal "hands" exploring their surrounding environment by means of their "fingers" (filopodia), one could say that the off-track GCs resemble "clenched hands," while the on-track GCs look like "open hands" with flat fingers extending on top of the substrate.

From the individual profiles, the mean GC heights were obtained (for details, see Materials and Methods), and their distribution is reported in Fig. 5(e). The analysis and Gaussian fits to the histogram data points clearly show that two distinct populations of GCs exist, which precisely correspond to the two GC groups of on-track and off-track axons + GCs identified in Fig. 5(c) and (d). Finally, the mean height [i.e., the centers of the Gaussian distributions in Fig. 5(e)] of the two groups of GCs was calculated from the respective data point set $(n=11$ and $n=10$ for the off-track and the on-track set, respectively), and are given along with the standard errors in Fig. 5(f). The statistical analysis showed that the difference in height of the two groups of GCs is highly significant $(p<0.01$, one-way ANOVA).

\section{Atypical Axon Growth behavior on the Patterned Surfaces}

Although we identified specific characteristics of the axonal growth driven by the presence of L1 patterns, we occasionally found abnormal situations with respect to what was described in the previous paragraph (see Fig. 6). The analysis of these particular cases provided an important guidance in interpreting a variety of phenomena associated with axonal growth.

In Fig. 6(a), a GC sits on a stripe of L1 for a relatively short time, as it can be deduced from the very short portion of the axon lying on the pattern. A similar case is also shown in Fig. 2(c). These GCs have a shape that is prominent with respect to the central part of the axon and, although effectively located onto the adhesion molecules pattern, they resemble GCs in the offtrack-like group. This behavior was detected in roughly $5 \%$ of all
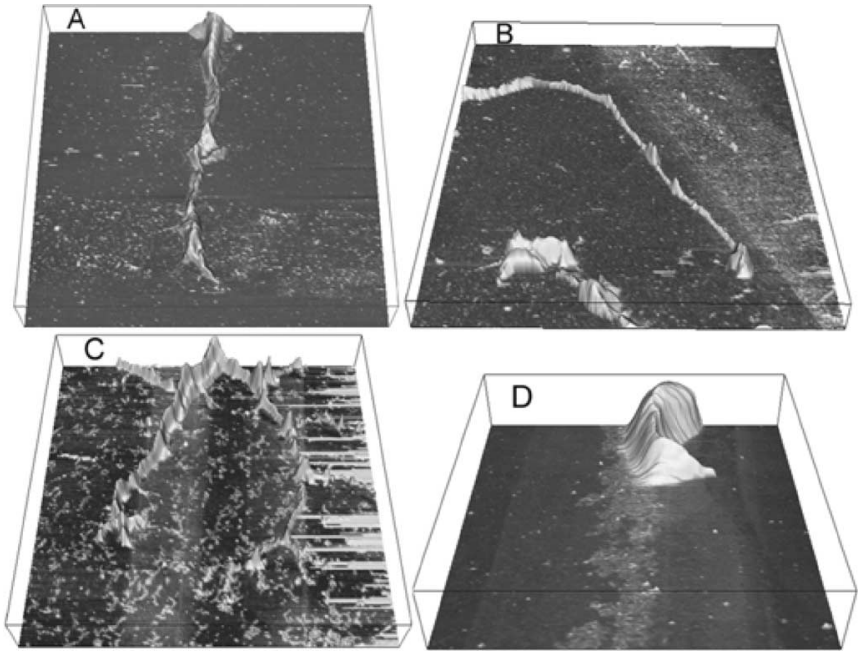

Fig. 6. Axons and GCs with atypical behavior. (a) GC that just entered a L1-coated area yet retains the prominent shape typical of off-track GCs. (b) Axon that grew parallel to an L1 stripe, but outside it, exhibits the prominent shape typical of off-track GCs. (c) Double track, due to defective $\mu \mathrm{CP}$, induced a bifurcation of the axon. (d) Axon grown onto an L1 stripe with irregular height and side edges, retains a steep and prominent shape typical of off-track GCs.

the imaged cases. This observation indicates that the substrateinduced modification in GC morphology appears only after a certain period of time, necessary for the GC to adapt its shape to reach the favorite conformation in the new environment. This characteristic time should correlate with studies of actin treadmilling and engorgement stage of axon outgrowth.

Another atypical behavior is shown in Fig. 6(b). In this image, an example is reported of an axon that runs parallel to the pattern without reaching it. Yet the change in direction of the axon growth when the GC came in the vicinity of the L1 track suggests that the neurite has felt the presence of the pattern. This effect could be due to traces of PDMS stamp contact that were not clearly detectable by AFM [see discussion of Fig. 6(b) next].

Atypical GC behaviors are also observed in areas of defective or incomplete L1 microprinting [see Fig. 6(c) and (d)].

Such areas were very rarely found in the middle of the stamps but comparatively often (up to $20 \%$ of the processes) in peripheral regions of the transferred pattern (e.g., the outmost $100 \mu \mathrm{m}$ close to the pattern borders). In Fig. 6(c), an imperfect transfer in the printing procedure of the protein A originated multiple tracks of L1. In Fig. 6(d), the L1 track broadened and spread over various heights, probably due to the slip of the PDMS stamp during the printing procedure. In these areas, GCs were in most cases characterized by prominent shape and steep edges, as if they were not growing onto an L1-coated surface [see Fig. 6(c) and (d)], suggesting the need of a uniform adhesion surface in order for the GC to assume the flat, propulsive shape.

\section{E. Conclusion}

In this paper, we studied the 3-D profile of axons and GCs of primary hippocampal neurons grown onto PDL surfaces patterned with L1. The L1 pattern specifically attracted the axonal 
network, while leaving the distribution and growth of neuronal soma and dendrites unaffected. 3-D imaging of neuronal processes allowed identifying specific behaviors of axons and GCs depending on the underlying molecular substrate. Axons became flatter with time in vitro, and this effect was more intense for axons growing on L1 stripes than for off-track axons, particularly at later stages in culture.

This effect became more dramatic when the analysis was concentrated on the GC. GCs onto L1 exhibited highly dynamic outgrowth, and flattened on the surface, in an attempt to maximize the sensory exploration of the environment and extend the advancing edge. On the other hand, GCs grown onto a permissive, but not attracting substrate, such as PDL, assumed a prominent and steep distal profile with a higher height-to-width ratio.

In conclusion, the association between functional behavior and morphological properties suggests that the terminal shape and height of the central domain of the GC are a function of the affinity for the substrate. The shape changes are likely to be associated with changes in actin treadmilling and related internal processes regulating protrusion, engorgement, and consolidation of axons. Further studies are in progress on living neurons with AFM to provide a more sensitive functional characterization, along with measurements of the mechanical properties that underliethe observed morphological changes.

\section{ACKNOWLEDGMENT}

The authors would like to thank Dr. M. Fanciulli (Laboratorio Nazionale MDM CNR-INFM, Agrate Brianza, Milan, Italy) for providing the silicon masters used for microprinting, Dr. P. Doherty for the generous gift of the L1-Fc plasmids, Dr. A. Diaspro (LAMBS Laboratories, Genova, Italy) for the use of the confocal facility, and Dr. M. Pesce for his expert technical assistance on the confocal microscope setup.

\section{REFERENCES}

[1] A. B. Huber, A. L. Kolodkin, D. D. Ginty, and J. F. Cloutier, "Signaling at the growth cone: Ligand-receptor complexes and the control of axon growth and guidance," Annu. Rev. Neurosci., vol. 26, pp. 509-563, 2003.

[2] E. W. Dent and F. B. Gertler, "Cytoskeletal dynamics and transport in growth cone motility and axon guidance," Neuron, vol. 40, pp. 209-227, 2003.

[3] J. R. Bamburg, "Introduction to cytoskeletal dynamics and pathfinding of neuronal growth cones," J. Histochem. Cytochem., vol. 51, pp. 407-409, 2003.

[4] M. Tessier-Lavigne and C. S. Goodman, "The molecular biology of axon guidance," Science, vol. 274, pp. 1123-1133, 1996.

[5] F. S. Walsh and P. Doherty, "Neural cell adhesion molecules of the immunoglobulins superfamily: Role in axon growth and guidance," Аnnu. Rev. Cell. Dev. Biol., vol. 13, pp. 425-456, 1997.

[6] R. O. Hynes and A. D. Lander, "Contact and adhesive specificities in the associations, migrations, and targeting of cells and axons," Cell, vol. 68, pp. 303-322, 1992.

[7] J. Brown and P. C. Bridgman, "Role of Myosin II in axon outgrowth," $J$. Hystochem. Cytochem., vol. 51, pp. 421-428, 2003.

[8] F. Mann and R. Geneviève, "Mechanisms of axon guidance: Membrane dynamics and axonal transport in semaphotin signalling," J. Neurochem., vol. 102, pp. 316-323, 2007.

[9] P. J. Meberg and J. R. Bamburg, "Increase in neurite outgrowth mediated by overexpression of actin depolymerization factor," J. Neurosci., vol. 20, pp. 2459-2469, 2000.
[10] J. E. Bear, T. M. Svitkina, M. Krause, D. A. Schafer, J. J. Loureiro, G. A. Strasser, I. V. Maly, O. Y. Chaga, J. A. Cooper, G. G. Borisy, and F. B. Gertler, "Antagonism between Ena/VASP proteins and actin filament capping regulates fibroblast motility," Cell, vol. 109, pp. 509-521, 2002.

[11] T. J. Diefenbach, V. M. Lathman, D. Yimlamai, C. A. Liu, I. M. Herman, and D. G. Jay, "Myosin 1c and myosin IIB serve opposing roles in lamellipodial dynamics of the neuronal growth cone," J. Cell Biol., vol. 158, pp. 1207-1217, 2002.

[12] K. Kalil and E. W. Dent, "Touch and go: Guidance cues signal to the growth cone cytosckeleton," Curr. Opin. Neurobiol., vol. 15, pp. 521-526, 2005.

[13] M. Matsuzawa, T. Tabata, W. Knoll, and M. Kano, "Formation of hippocampal synapses on patterned substrates of a laminin-derived synthetic peptide,” Eur. J. Neurosci., vol. 12, pp. 903-910, 2000.

[14] K. Ganguly, L. Kiss, and M. Poo, "Enhancement of presynaptic neurona excitability by correlated presynaptic and postsynaptic spiking," Nat. Neurosci., vol. 3, pp. 1018-1026, 2000.

[15] A. K. Vogt, G. Wrobela, W. Meyerb, W. Knoll, and A. Offenhausser "Synaptic plasticity in micropatterned neuronal networks," Biomaterials, vol. 26, pp. 2549-2557, 2005.

[16] A. K. Vogt, L. Laue, W. Knoll, and A. Offenhäusser, "Micropatterned substrates for the growth of functional neuronal networks of defined geometry," Biotechnol. Prog., vol. 19, pp. 1562-1568, 2003.

[17] A. Offenhäusser and A. K. Vogt, "Defined networks of neuronal cells in vitro," in Nanobiotechnology, C. Niemeyer and C. Mirkin, Eds. Weinheim, Germany: Wiley, 2004, pp. 66-76.

[18] D. Cojoc, F. Difato, E. Ferrari, R. B. Shahapure, J. Laishram, M. Righi, E. M. Di Fabrizio, and V. Torre, "Properties of the force exerted by filopodia and lamellipodia and the involvement of cytoskeletal components," PLoS ONE, vol. 2, p. e1072, 2007.

[19] H. Sorribas, C. Padeste, and L. Tiefenauer, "Photolithographic generation of protein micropatterns for neuron culture applications," Biomaterials, vol. 23, pp. 893-900, 2002.

[20] D. Stenger, C. J. Pike, J. J. Hickman, and C. W. Cotman, "Surface determinants of neuronal survival and growth on self-assembled monolayers in culture," Brain Res., vol. 630, pp. 136-147, 1993.

[21] B. C. Wheeler, J. M. Corey, G. J. Brewer, and D. W. Branch, "Microcontact printing for precise control of nerve cell growth in culture," $J$ Biomech. Eng., vol. 121, pp. 73-78, 1999.

[22] G. M. Whitesides, E. Ostuni, S. Takayama, X. Jiang, and D. E. Ingber "Soft lithography in biology and biochemistry," Апnи. Rev. Biomed. Eng., vol. 3, pp. 335-373, 2001.

[23] A. Folch and M. Toner, "Microengineering of cellular interactions," Аnnu. Rev. Biomed. Eng., vol. 2, pp. 227-256, 2000.

[24] A. A. Oliva, Jr., D. J. Conrad, C. E. Kingman, H. G. Craighead, and G. A. Banker, "Patterning axonal guidance molecules using a novel strategy for microcontact printing," Neurochem. Res., vol. 28, pp. 1639-1648, 2003.

[25] H. Kamiguchi and V. Lemmon, "Neural cell adhesion molecule L1: Signaling pathways and growth cone motility," J. Neurosci. Res., vol. 49, pp. 1-8, 1997.

[26] M. Moos, R. Tacke, H. Scherer, D. Teplow, K. Fruh, and M. Schachner "Neural adhesion molecule L1 as a member of the immunoglobulin superfamily with binding domains similar to fibronectin," Nature, vol. 334, pp. 701-703, 1988.

[27] A. N. Van Den Pol and W. T. Kim, "NILE/L1 and NCAMpolysialic acid expression on growing axons of isolated neurons," J. Comput. Neurol., vol. 332, pp. 237-257, 1993.

[28] M. A. Silverman, S. Kaech, M. Jareb, M. A. Burack, L. Vogt, P Sonderegger, and G. Banker, "Sorting and directed transport of membrane proteins during development of hippocampal neurons in culture," Proc. Nat. Acad. Sci. USA, vol. 98, pp. 7051-7057, 2001.

[29] V. Lemmon, K. L. Farr, and C. Lagenaur, "L1-mediated axon outgrowth occurs via a homophilic binding mechanism," Neuron, vol. 2, pp. 1597 $1603,1989$.

[30] G. Kadmon and P. Altevogt, "The cell adhesion molecule L1: Speciesand cell-type-dependent multiple binding mechanisms," Differentiation, vol. 61, pp. 143-150, 1997.

[31] J. Q. Davis and V. Bennet, "Ankyrin binding activity shared by the neurofascin/L1LNrCAM family of nervous system cell adhesion molecules," J. Biol. Chem., vol. 269, pp. 27163-27166, 1994.

[32] J. J. Langone, "Protein A of Staphylococcus aureus and related immunoglobulin receptors produced by streptococci and pneumococci," Adv. Immunol., vol. 32, pp. 157-252, 1982.

[33] A. Alessandrini and P. Facci, "AFM: A versatile tool in biophysics," Meas. Sci. Technol., vol. 16, pp. R65-R92, 2005. 
[34] T. Hosono, M. Yamanaka, T. Tojima, Y. Yamane, H. Sadamoto, D. Hatakeyama, H. Haga, K. Kawabata, K. Abe, and E. Ito, "Atomic force microscopic observation of three-dimensional morphological changes of neurons when stimulated by a neurotransmitter," Jpn. J. Appl. Phys., vol. 39, pp. 3940-3945, 1999.

[35] H. McNally and R. B. Borgens, "Three-dimensional imaging of living and dying neurons with atomic force microscopy," J. Neurocytol., vol. 33, pp. 251-258, 2004.

[36] H. McNally, "Imaging and manipulating living neurons with atomic force microscopy," presented at 26th Annu. Int. Conf. IEEE EMBS, San Francisco, CA, Sep. 1-5, 2004.

[37] H. McNally, B. Rajwa, J. Sturgis, and J. P. Robinson, "Comparative threedimensional imaging of living neurons with confocal and atomic force microscopy," J. Neurosci. Methods, vol. 142, pp. 177-184, 2005.

[38] B. A. Smith, H. Roy, P. De Koninck, P. Grütter, and Y. De Koninck, "Dendritic spine viscoelasticity and soft-glassy nature: Balancing dynamic remodeling with structural stability," Biophys. J., vol. 92, pp. 1419-1430, 2007.

[39] D. Ricci, M. Grattarola, and M. Tedesco, "Growth cones of living neurons probed by atomic force microscopy," Methods in Molecular Biology, Atomic Force Microscopy Biomedical Methods and Applications, Totowa, NJ: Humana Press, 2004.
[40] R. Lal, B. Drake, D. Blumberg, D. R. Saner, P. K. Hansma, and S. C. Feinstein, "Imaging real-time neurite outgrowth and cytoskeletal reorganization with an atomic force microscope," Amer. J. Physiol., vol. 269, pp. 275-285, 1995.

[41] V. Parpura, P. Haydon, and E. Henderson, "Three-dimensional imaging of living neurons and glia with the atomic force microscope," J. Cell Sci., vol. 104, pp. 427-432, 1993.

[42] P. Doherty, E. Williams, and F. S. Walsh, "A soluble chimeric form of the L1 glycoprotein stimulates neurite outgrowth," Neuron, vol. 14, pp. 5766, 1995.

[43] D. L. Simmons, "Dissecting the modes of interactions amongst cell adhesion molecules," Dev. Suppl., pp. 193-203, 1993.

[44] G. A. Banker and K. Goslin, Culturing Nerve Cells. Cambridge, MA: MIT Press, 1998.

Author's photographs and biographies not available at the time of publication. 